\title{
EL TESTAMENTO DE JUAN MOLINER (†1403): UN FÍSICO AL SERVICIO DE LA MONARQUÍA NAVARRA DURANTE LA BAJA EDAD MEDIA*
}

\author{
Fernando Serrano Larráyoz
}

Universidad de Alcalá

\section{RESUMEN}

A través del testamento de Juan Moliner, médico de los reyes Carlos II y Carlos III de Navarra, el presente estudio pretende acercarse a su persona, familia, ámbito social y nivel socioeconómico en el que desarrolla su vida. Los datos que aporta dicho documento arrojan algo de luz sobre su ciclo vital, hasta el momento bastante desconocido, pese a ser uno de los más importantes médicos cristianos que atendieron a Carlos III 'el Noble'. A todo esto hay que añadir la dimensión espiritual y religiosa de sus últimas voluntades, que vienen a completar la mentalidad y el modo de vida de este personaje.

PALABRAS CLAVE: Juan Moliner. Médico real. Testamento. Siglos XIV-XV.

\section{THE TESTAMENT OF JUAN MOLINER (†1403): A DOCTOR AT THE SER- VICE OF THE KINGS OF NAVARRE DURING THE LATE MIDDLE AGES}

\begin{abstract}
This work is an approach to the life and socioeconomic context of Juan Moliner (doctor of kings Carlos II and Carlos III of Navarre), based on the study of his testament. The document provides enlightening information about this figure, quite unknown despite being one of the most important Christian doctors at the service of Carlos III 'El Noble'. In addition, his last will has a religious and spiritual dimension that allows to have an overall perspective on the mentality and lifestyle of this historical character.
\end{abstract}

KEY WORDS: Juan Moliner. Royal doctor. Testament. $14^{\text {th }}-15^{\text {th }}$ centuries.

* Este trabajo ha sido realizado en el marco del proyecto de investigación FFI2011-29117C02-01 concedido por el Ministerio de Ciencia e Innovación (ahora Ministerio de Economía y Competitividad). Agradezco los comentarios y sugerencias a Fermín Miranda García, Juan Jesús Virto Ibáñez, Jon Arrizabalaga Valbuena y Carmel Ferragud Domingo. Del mismo modo, agradezco a Izaskun Aisa Larumbe e Itziar Zabalza Aldave su ayuda en las dudas de transcripción del testamento incluido al final del estudio. 


\section{INTRODUCCIÓN}

El testamento de Juan Moliner, - uno de los físicos o médicos cristianos más cercanos a la familia real durante los reinados de Carlos II y Carlos IIIpermite aproximarse a su persona, a su nivel de vida y a su dimensión espiritual y religiosa. El de un hombre concreto que sirvió a los monarcas navarros entre el último cuarto de siglo XIV e inicios del XV. De hecho, la práctica testamentaria no solo se ha revelado como el mejor de los testimonios para conocer los sentimientos íntimos de cada individuo ante la muerte, sino que también es reflejo de la situación socioeconómica y familiar del testador al final de su vida. Ni qué decir tiene, como afirman Julia Pavón Benito y Ángeles García de la Borbolla que, «el testamento, sobre todo en los siglos XIV y XV, se configura como un acto de previsión ante las trascendentales consecuencias que repercuten directamente en el destino eterno del alma». En este caso, sin dejar de lado las consideraciones espirituales que el propio testamento implica, se pretende ahondar más concretamente en la faceta referida a la repercusión social y familiar alcanzada por el difunto en vida e intentar comprender la trayectoria vital de maestre Juan Moliner. Un planteamiento - el de la actitud ante la muerte a través de las mandas testamentarias - que no resulta novedoso puesto que recientemente el estudio de los testamentos y sus implicaciones en la mentalidad de los navarros de los últimos siglos de la Edad Media ya ha sido tratado por algunos especialistas ${ }^{1}$. No obstante, debido a la escasez de este tipo de documentación sobre aquellos que se dedicaban a la práctica del cuidado de la salud, más concretamente los médicos vinculados al ámbito cortesano navarro, cobra una especial importancia ${ }^{2}$.

1 Sin ánimo de ser exhaustivo, tan solo mencionar los estudios de Baldó Alcoz, 2005, y de Pavón Benito y García de la Borbolla, 2007, en los cuales se ofrece una amplia bibliografía y estado de la cuestión sobre Navarra.

2 Referencias a testamentos sobre profesionales sanitarios durante el periodo bajomedieval en Navarra, pueden consultarse en Baldó Alcoz, García de la Borbolla y Pavón Benito, 2005, pp. 155-226. Quien haya leído el libro Morir en la Edad Media (Pavón Benito y García de la Borbolla, 2007) podría criticarme el no estudiar en este mismo trabajo el testamento de Miguel de Mares, considerado como médico de los reyes Carlos II y Carlos III de Navarra. Sin embargo, dicho Miguel jamás fue médico. Durante buena parte de su vida ejerció de consejero real hasta su fallecimiento en 1406: a present morador en'l Burgo de Sant Saturnin de Pampeluna et fue del dicho Miguel nascido en Carenten, sobre la mar en Normandia (Archivo Parroquial de San Saturnino (=APSS), caj. 1, Pergaminos, $\mathrm{n}^{\circ} 11$, sec. I, $n^{\circ}$ ant. 38 (12)). El error puede venir dado porque en la ficha manuscrita, catalogada por el profesor Santos García Larragueta y conservada en el propio archivo lo menciona como médico, aunque en ningún momento se hace alusión a esta actividad, sino como clérigo y consejero del rey. 


\section{APUNTES BIOGRÁFICOS DE JUAN MOLINER Y SU FAMILIA}

Se desconocen los orígenes de Juan Moliner, cuyas primeras referencias datan del año 1377, y cómo llegó al servicio de Carlos II y del infante Carlos, futuro Carlos $\mathrm{III}^{3}$. Tras el fallecimiento del primero y el ascenso al trono de su hijo se llevaron a cabo una serie de reformas en la organización de la Administración y de la propia Casa Real, que, como no podía ser de otro modo, con el tiempo afectaron al propio físico. De mayo de 1388 es su nombramiento como médico del rey, siéndole asignados 200 florines trimestrales en concepto de gajes para su mantenimiento, el de dos servidores y tres caballos ${ }^{4}$. Un salario que se suma a los 300 florines (360 libras) de pensión anual que le habían sido asignados, a recibir sobre el almudí de Tudela, tasado en 250 libras, y sobre la pecha del val de San Esteban, en la merindad de Estella, estimada en 110 libras:

A maestre Johan Moliner, fisigo del rey, al quoall el dicho seynnor ordeno que ouiesse de pension a recebir en la su cambra a los dineros en cada un aynno, segunt se contiene por dos letras del dicho seynnor dadas el primero dia de mayo et $\mathrm{VI}^{\circ}$ de marco anno LXXXVIII, 300 florines, et de si el dicho seynnor queriendo fazer como eill sia bien pagado de la dicha pension et assin que meyor et mas diligentement sierua et sia tenido de seruir le ha dado en paga de los dichos 300 florines que, ha 24 sueldos pieca, valen 360 libras; el almudi de Tudela (...) en precio de 250 libras cad'aynno, valgan mas o menos, et sobre la pecha de val de Sant Esteuan, de la merindat d'Esteilla (...) 110 libras $(\ldots)^{5}$.

Unos importantes ingresos que vienen a complementarse con otras generosas aportaciones que se le van entregando a lo largo de su vida por parte del

3 Archivo General de Navarra (=AGN), Comptos. Documentos, caj. 30, $\mathrm{n}^{\circ}$ 68, 1. Hay quien afirma que tenía el título de maestro en artes y bachiller en medicina (Narbona Cárceles, 2006, p. 255). No obstante, por mi parte no he encontrado dato alguno que corrobore estudios universitarios, aunque parece lo más probable. Si aceptamos que el título de 'maestre' es reflejo, en los físicos cristianos, de un título académico universitario, la gran mayoría de quienes sirvieron en la corte navarra debieron de haber pasado por la enseñanza superior, pero no parece que sea así. Por el contrario, el título de 'maestre' aplicado a un judío implicaba una distinción honorífica, concomitante con la dignidad profesional equivalente, pero no puede ser interpretado como grado académico. De hecho estos últimos tenían prohibido el acceso a la Universidad, con lo cual es imposible que la denominación de 'maestre' refleje su paso por las aulas (Serrano Larráyoz, 2004, p. 113). Sobre la formación de los sanadores hebreos -pero también de cristianos y musulmanes-, véase García Ballester, 2001, pp. 213-225.

4 AGN, Comptos. Documentos, caj. 57, no 39 y caj. 57, no 40, 3 .

5 AGN, Comptos. Registros, no 209, fol. 57v. 
monarca, tanto en dinero como en vestiduras, para cubrir sus necesidades o como agradecimiento especial por sus servicios ${ }^{6}$. Reflejo de su buen hacer en el cuidado de la familia real y de algunos de sus servidores que requirieron de sus saberes, como Andrés Dehan (Serrano Larráyoz, 2004, p. 132)7.

Muy poco es lo que se conoce respecto a la vida personal de Juan Moliner. Realmente, si se dejan de lado sus fructíferas actividades sanitarias, casi todos los datos sobre su familia, algunas de sus relaciones personales, bienes muebles o creencias personales pueden encontrarse en su testamento, fechado en Olite el 13 de febrero de $1403^{8}$. Así, desconocemos cuándo contrajo matrimonio con Gracia de Ursúa9, con la que tuvo dos hijos, Peyretón y Lancelot, y una hija, Juana, la más joven de los tres probablemente. Al día siguiente de la muerte del físico el monarca concede a los dos varones, bajo la tutela de su madre, 50 cahíces de trigo anuales, asignados sobre las pechas de Oteiza y Añézcar - que ya recibía su padre - para que puedan ser criados et aprender sciencia. Unos privilegios aumentados al año siguiente y que debían continuar hasta que alcanzaran la hedat de 24 aynnos $^{10}$. En ningún momento se alude a una hija, quizás porque se trata de un privilegio destinado a aquellos en que se confía que puedan continuar el oficio de su padre - no olvidar las restricciones que tenían las mujeres de aquella época para ejercer la medicina ${ }^{11}$ - De hecho en las

6 Como en el caso de los paños que recibió junto con Juan des Bordes, barbero del rey, por el servicio que le prestaron sangrándole el codo (AGN, Comptos. Documentos, caj. 71, no 42, 6).

7 AGN, Comptos. Documentos, caj. 57, $\mathrm{n}^{\mathrm{o}} 72,15$. Andrés Dehan aparece documentado como maestre del Hostal del rey (1387) y como consejero del rey y alcaide del castillo de Tudela (1388) (AGN, Comptos. Documentos, caj. 52, nº 28, 1 y AGN, Comptos. Documentos, caj. 53, no 34,39$)$.

8 APSS, caja 1, Pergaminos, $\mathrm{n}^{\circ} 7$, sec. I, $\mathrm{n}^{\circ}$ ant. 34 (8).

9 En 1406 y 1411 se documenta que tenía un hermano llamado Íñigo García de Ursúa (AGN, Comptos. Documentos, caj. 82, $\mathrm{n}^{\circ}$ 9, 19 y caj. 106, $\mathrm{n}^{\circ}$ 1, 7). En 1406 ella misma se denomina Gracia Bastán (Baztán?), viuda, muger qui fue de maestre Johan Molliner, fisico qui fue del seynor rey. Es probable que ambos pertenecieran a alguna rama del linaje de los Ursúa, de la que algunos de sus miembros fueron castellanos de San Juan de Pie de Puerto (Ramírez Vaquero, 1990, p. 114). En enero de 1384 un tal Íñigo de Ursúa, escudero, reconoce haber recibido del recibidor de Ultrapuertos 30 libras de carlines prietos, asignados sobre el 'francaje' de Mixa y Ostabarets (AGN, Comptos. Documentos, caj. 45, $\mathrm{n}^{\mathrm{o}} 16,11$ ).

10 AGN, Comptos. Documentos, caj. 89, n 10, 2.

11 Está comprobado que entre finales del siglo XIV y principios del XV, el ejercicio de la medicina general -no solo el ginecológico- también era practicado por algunas mujeres. Desde el punto de vista social, no eran curanderas, pues su práctica estaba permitida por los organismos correspondientes de control profesional, como ocurre en la Corona de Aragón. Sin embargo, la práctica legal de la medicina llevada a cabo por mujeres se vio interrumpida con 
disposiciones testamentarias de Juan Moliner no se considera necesario que la hija tenga la misma educación (leer y escribir) que sus hermanos, sino tan solo el conocimiento de las buenas costumbres, con vistas a conseguir un esposo lo suficientemente importante con el que fortalecer su linaje. Moliner señala que, una vez fallecida su mujer — Gracia de Ursúa-, los hijos vayan heredando por orden de edad y prioridad de sexos:

(...) l'uno por muert del otro por herencia legitima et mayorio segunt fuero de Navarra. Et en caso que el dicho Peyreton, mi fijo, en su vida o su criazon enpues su muert o el dicho Lançalot, mi fijo, enpues sus dias o su criazon enpues eill [o la] dicha Johana, mi fija, o sus criazones o quoalquiere d'ellos o de sus genoillas (...).

Establece la condición de no poder vender ni enajenar su herencia, salvo la mitad de los bienes muebles correspondientes a su esposa, cuyo disfrute ésta podía ejercer en vida como quisiera. Lo cierto es que Juana Moliner sobrevivió a sus dos hermanos ${ }^{12}$ y heredó los bienes de su padre vinculados a mayo-

la progresiva clericalización de la cultura (Martínez Crespo, 1994, pp. 44-45). La imposibilidad de acceso a la formación universitaria para las mujeres, y la posterior prohibición del ejercicio médico para aquellas y aquellos no licenciados o no examinados por un tribunal autorizado, ha llevado a diferentes interpretaciones sobre el por qué de la prohibición de la formación universitaria y de la práctica de las mujeres. Así, mientras hay quien considera que es un ejemplo de discriminación social por razón de sexo (Green, 1989, p. 447); otros creen que el objetivo de esta regulación no eran las mujeres por el hecho de ser mujeres sino por tratarse de empíricas (McVaugh, 1993, pp. 103-107). Sobre esto también hay quien piensa que tal marginación está motivada por intentar mantener vías alternativas de obtener los conocimientos. Unas vías alternativas que se encontraban fuera de lo académico y fuera de la palabra escrita, vinculadas con el platonismo - que cuestionaban el aristotelismo como soporte del mundo académico medieval-, y que es fundamental para entender las formas del conocimiento místico mayoritariamente femenino. La negativa a permitir la práctica médica a quienes no estuvieran examinados por un tribunal se debió a la necesidad de impedir la existencia de fórmulas de establecimiento de autoridad fuera de las negociadas desde el poder establecido (Salmón Múñiz, 1997, pp. 38-43). Sobre estos aspectos puede consultarse también el trabajo de Cabré i Pairet y Salmón Múñiz, 2001, pp. 55-76. Con todo, la presencia de mujeres sanadoras de alto nivel queda constatada, pese a ser casos minoritarios, en una tal Doza, fisica del conde de Foix, que trató, en 1403, junto con otros físicos y cirujanos de prestigio al rey Carlos III el 'Noble' de Navarra (Serrano Larráyoz, 2004, p. 78); y, a finales del siglo XV, en la física Mencia González, en Aranda de Duero (López Gómez, 2010, pp. 215-233).

12 La última referencia sobre Peyretón y Lancelot localizada es de diciembre de 1411, cuando su madre reconoce, en nombre de ambos, que ha recibido los provechos y emolumentos de un molino en el puente de Tudela (AGN, Comptos. Documentos, caj. 106, $\mathrm{n}^{\circ}$ 1, 17). Dichos beneficios ya habían sido concedidos por el monarca a su padre años antes (AGN, Comptos. Documentos, caj. 59, $\mathrm{n}^{\mathrm{o}} 7$ ). 
razgo o mayorio ${ }^{13}$. Casó con Juan de Itúrbide, notario de la Cámara de Comptos, y tuvo un hijo llamado como su padre y abuelo. No obstante, la muerte de ésta antes que su esposo planteó algunos problemas sobre la herencia.

El testamento de Itúrbide -7 de abril de 1457- informa que su hijo quedaua fuera de su seso y en frenesia de locura, por lo que no tardó en revisar las cláusulas del testamento de su suegro, en el que dejaba expresamente claro que su herencia debía ser entregada a los sucesores de sus hijos, salvo que ninguno d'eillos fuese loco, gastador o de mal regimiento o gouernamiento. Así, habiendo fallecido los otros hijos que tuvo con Juana Moliner, por descargo de su conciencia, decidió cumplir la última voluntad de Juan Moliner sobre la fundación de una capellanía anual perpetua simple con las rentas de los bienes a heredar en caso de no existir descendencia directa, tal y como se verá más adelante ${ }^{14}$. Por este motivo ordenará a sus albaceas o cabezaleros que mantengan a su hijo con las rentas de sus propios bienes, mientre el fuere biuo ${ }^{15}$.

Tampoco es demasiado lo que se sabe sobre los bienes muebles que posee maestre Moliner al final de su vida. No debieron resultar pocos, aunque su testamento no es demasiado explícito. Por un lado concede a su mujer la casa que posee en la Rua maor d'la Correyeria de Pomplona con su mueble (actualmente correspondería con el tramo de la Calle Mayor más próxima a la iglesia de San Lorenzo) ${ }^{16}$, casa adquirida junto con otras, probablemente en 1398, al maestro de la moneda del rey ${ }^{17}$,

13 El mayorazgo es una forma de propiedad vinculada en la que el titular dispone de la renta, pero no de los bienes que la producen, a la que se accede mediante un orden sucesorio prefijado. Así, entre los miembros del estamento nobiliario, el patrimonio cobró una especial significación, debido a que tanto para su propia subsistencia como para el mantenimiento de su prestigio socioeconómico y su poder político, dependían fundamentalmente de las rentas que aquél generaba. Además, a medida que fue desarrollándose en la mentalidad colectiva de la nobleza la imagen y conciencia del linaje, el patrimonio pasó a constituir no ya la fortuna personal de cada uno de sus miembros, sino la del propio linaje (Noáin Irisarri, 2003, pp. 9396). En el caso concreto que estamos tratando, pese a que Juan Moliner no pertenece a la nobleza, el interés por preservar de la ruina a sus herederos, como consecuencia de una mala gestión del patrimonio, contribuye al desarrollo del linaje, en tanto que la idea de la partición hereditaria lo destruye. Sobre esto, véase Noáin Irisarri, 2003, pp. 97-98.

14 Véase nota $\mathrm{n}^{\mathrm{o}} 39$.

15 APSS, caj. 2, Pergaminos, $\mathrm{n}^{\circ} 33$, sec. I, $\mathrm{n}^{\circ}$ ant. 64 (32).

16 El nombre de la calle se debía al gremio de 'corregers' o corredores de comercio (Martinena Ruiz, 1974, p. 207). En catalán 'corretger' es el que trabajaba el cuero (corret$\mathrm{ja}=$ correa). Agradezco el dato a Carmel Ferragud Domingo.

17 AGN, Comptos. Registros, no 243, fol. 79r. 
la quoal se afruenta d'una part con casas de Martin Bertran, mercadero, vezino de Pomplona, et d'la otra part con casas de Miguel d'Eguaras, correyero, vecino de Pomplona, et todos et quoalesquiere otros bienes muebles, heredamientos pertenecientes a mi, asi como casas, casales, piezas, vinas, huertos, olivares, prados et vezindades o qualesquiere otros bienes, heredamientos a mi en todo el regno de Nauarra o en quoalquiere otra tierras et seynnorios sean et puedan ser et pertenescer por quoalquiere titulo o razon sean (...).

Sobre la ubicación y posesión concreta de algunos bienes raíces queda la información de la distribución que hace entre sus hijos e hija, como una arinzada $\left(898,2 \mathrm{~m}^{2}\right)$ de viña que deja a Peyretón en Cascailleta, de Pamplona, otra arinzada en Mutiloa ${ }^{18}$ que dona a Lancelot, y otra arinzada que deja a Juana en el termino de Sayquin ${ }^{19}$, pero poco más.

De lo dicho hasta ahora puede comprobarse que la situación económica de la familia Moliner era más o menos holgada. Tanto es así que de las deudas que deja recogidas en su testamento y que no ha cobrado en el momento de redactar sus últimas voluntades, dan la sensación que en más de una ocasión ejerce como prestamista entre distintos personajes, unos con más renombre que otros, pero casi todos con una posición social distinguida, como Pedro de la Plana, molinero de Tudela ${ }^{20}$, que le debía 200 libras «febles»; el fallecido Martín Ibáñez de Los Arcos, notario, y Miguel de Eguaras, correyero y uno de sus vecinos de Pamplona, deudores de 2 escudos de oro respectivamente; Pascual de Lizarraga y Juan de Sangüesa, el mercader y notario de la Cort, ambos vecinos de Estella, que también le debían 600 libras «febles» y parte de 220 libras fuertes respectivamente; Jacques du Tronchoy, llamado Tronchet, escudero ${ }^{21}$, y su hermano Francés, que le adeudaban un escudo cada

18 Se empleó esta forma para designar indistintamente a las localidades de Mutilva Alta y Mutilva Baja (Gran Enciclopedia de Navarra, t. VIII, p. 10).

19 Este término aparece documentado en Mendillorri y Mutilua Alfa (APSS, caj. 2, Pergaminos, $\mathrm{n}^{\mathrm{o}} 33$, sec. $\mathrm{I}, \mathrm{n}^{\mathrm{o}}$ ant. $\left.64(32)\right)$.

20 Probablemente la relación con el molinero esté en relación con las rentas que Juan Moliner cobraba sobre varias ruedas de molino en el puente de Tudela. Así, en febrero de 1391, el rey le concede la mitad de la segunda casa o molino de dicho puente, con todos los derechos (AGN, Comptos. Documentos, caj. 59, $\mathrm{n}^{\mathrm{o}} 7$ (1)). Al día siguiente de su fallecimiento el monarca se la concede a sus hijos (AGN, Comptos. Documentos, caj. 89, $\mathrm{n}^{\circ} 11$ ). Años después, en diciembre de 1405, Sancho de Aoiz, abad de Urroz y uno de los albaceas del testamento, entrega a tributo y alquiler una rueda de molino al molinero de Tudela Juan de Andosilla, para su disfrute durante el año siguiente (AGN, Comptos. Documentos, caj. 81, $\mathrm{n}^{\mathrm{o}} 12,5$ ).

21 En 1378 es aludido como valet de cámara del rey Carlos II (AGN, Comptos. Documentos, caj. 33, $\mathrm{n}^{\mathrm{o}} 7,1$ ); en 1385 como almirante de la villa de Sangüesa (AGN, Comptos. 
uno; y los herederos de Juan García de Beunza ${ }^{22}$, deudores de 200 florines de oro de Aragón. Por lo que respecta a ciertas tazas de plata - no debe olvidarse el valor simbólico que la vajilla de lujo adquiría en una sociedad donde el sustento diario era para la gran mayoría su mayor preocupación, además del valor monetario que alcanzaban en caso de escasez de dinero- Juan Moliner menciona a Juan Cruzat, chambelán, y a la mujer de Pedro de $\mathrm{Arce}^{23}$, quienes le habían empeñado dos tazas respectivamente, y a Pedro de Acedo ${ }^{24}$, y Juan de Izco, especiero y mercader de Pamplona respectivamente, que le habían empeñado una cada uno.

El aprecio de Juan Moliner por sus criados es también considerable, dejando a su moza Marito 80 libras fuertes, pora si et pora su casamiento, que le debía el arcediano de val de Aibar, los quoales eill me los tiene d'la mi pension por el mi benefiçio deuido por los prior, capitol et canonigos de Pomplona. Le deja, además, 10 cahíces de trigo y 6 cargas de vino mosto del meior de mi casa. A García, otro mozo, le concede 3 cahíces y medio de trigo sobre dicho arcedianato, y a Micheto, otro de sus mozos, 3 florines de oro. No obstante, fue Enecot, su mozo y servidor personal, quien recibe la mayor demostración de afecto al serle concedida una hopalanda de peis sen la forradura y un capirote, el mejor, además de la mula morena que yo solia caualgar ante desta mi enfermedat y de permitirle vivir en su casa de Pamplona y de encomendarle el cuidado de sus hijos:

Documentos, caj. 49, $\mathrm{n}^{\circ} 73,6$ ); en 1388 como sargento de armas (AGN, Comptos. Documentos, caj. 57, $\mathrm{n}^{\circ}$ 40, 1); y en 1397 como alcaide del castillo de Falces (AGN, Comptos. Documentos, caj. 72, $\mathrm{n}^{\mathrm{o}} 30,36$ ).

22 En julio de 1386 se le menciona como mercader de Pamplona (AGN, Comptos. Documentos, caj. 52, $\mathrm{n}^{\circ}$ 51, 3); y en 1396 como recibidor de la merindad de Pamplona (AGN, Comptos. Documentos, caj. 78, $\mathrm{n}^{\circ}$ 5, 5). A principios de 1400 todavía vive, según notificación de una sentencia de la Cort, en un pleito que mantiene contra García de Lanz, vecino de Pamplona (AGN, Comptos. Papeles Sueltos, $2^{\mathrm{a}}$ Serie, leg. 8, $\left.\mathrm{n}^{\mathrm{o}} 40,25\right)$.

23 En 1400 es reconocido como zapatero de Pamplona, al que se le deben 11 libras y 8 sueldos fuertes por zapatos que realizó para el infante y las infantas (AGN, Comptos. Papeles Sueltos, $2^{\mathrm{a}}$ Serie, leg. $\left.4, \mathrm{n}^{\mathrm{o}} 174\right)$.

24 Boticario vinculado con Juan Moliner, a quien en numerosas ocasiones se le adquieren diversas medicinas para la familia real. Un ejemplo puede ser en [1387?], cuando Sancho de Ayanz reconoce que ha recibido medicinas de Pedro de Acedo, valoradas en 15 florines, para cierta enfermedad de Leonel, hijo bastardo del rey Carlos II, a quien tenía a su cuidado. El documento contiene la diligencia del mencionado Juan Moliner (AGN, Comptos. Documentos, caj. 173, $\mathrm{n}^{\circ} 13,5$ ) Sobre este personaje véase, Serrano Larráyoz, 2004, pp. 55, 89 y 192; y Serrano Larráyoz, 2011, pp. 278-279. 
(...) et mando mas que el dicho Enecot more et viua en toda la su vida en mi casa de Pomplona, comiendo et beuiendo de lo que en casa se trobara comunlment (sic), et asi bien que aya de auer a bien vista d'la dicha Gracia, mi muger, su bestir sufiçient segunt la facultat vista entre eilla et eill sera, et le acomendo al dicho Enecot las dichas mis criaturas que la regesta segunt fuesen suyas propias, et le encargo en la su anima et conciencia que renda a las dichas mis criaturas si algunas cosas en los tiempos pasados a tomado o apartado en los tiempos pasados de mis bienes.

Los recursos económicos dejados a sus hijos, además de las viñas anteriormente mencionadas, fueron 20 sueldos carlines a cada uno por fazer de aqueillos todas sus propias voluntades y 100 libras carlines a Juana y Lancelot respectivamente, para cuando contrajeran matrimonio. Más valor tienen para Juan Moliner los libros que posee sobre medicina, que yo a present he en Navarra et quoalesquiere otros libros que yo he de ciencia, que dona a Peyretón, su primogénito. Dos de estos libros le habían sido prestados por los franciscanos de Estella y debían ser devueltos. Añadir que también poseía alguno de nigromancia (saberes con los que se pretende incidir en el futuro invocando a los demonios) y que ordena quemar ${ }^{25}$, quizás por recomendación de su confesor, el franciscano fray Pedro de Eza, confesor también de la reina Leonor, y a quien dona cuatro florines de oro de Aragón ${ }^{26}$. Con todo, establece que los libros pasen a Lancelot en caso de que Peyretón non quisiere aprender en la dicha ciencia, dejando la posibilidad de que, si ninguno de sus hijos quería continuar sus pasos ${ }^{27}$, los libros fueran a parar a manos de su madre para que los guardara y vendiera

25 Sobre los libros, véase Serrano Larráyoz, 2009, pp. 548-549. La utilización de la nigromancia por parte de algunos médicos parece que no fue del todo infrecuente, véase el caso más tardío del judeoconverso Francisco López de Villalobos (Arrizabalaga, 2002, p. 34).

26 De origen castellano estuvo al servicio de la reina entre 1395 y 1406 (Marcotegui Barber, 2009, p. 92). Por lo que respecta a la presencia del confesor en el testamento, parece motivada por la especial influencia que llega a tener en el fiel al ser su director espiritual (Pavón Benito y García de la Borbolla, 2007, pp. 265-266). En este caso se hace evidente por la intercesión que a cambio le solicita por su donación: por tal que mi anima aya en comienda. Aunque difícil de precisar, es posible que Juan Moliner estuviera imbuido de la «espiritualidad renovada que desplegaron las órdenes mendicantes», a partir del siglo XIII, basada en la pobreza y la predicación, y cuya propuesta era cristianizar «unas actividades lucrativas que hasta entonces se había considerado absolutamente explotadoras y, por tanto, moralmente inaceptables». Unas premisas franciscanas que hace «pensar que una porción significativa de la elite de los médicos universitarios participó del nuevo ethos mendicante, y que sus preferencias se inclinaron preferentemente a la orden terciaria de los frailes menores» (Arrizabalaga, 2012 (en prensa)). Agradezco a su autor la posibilidad de consultar el texto original.

27 Se sabe que la flor y nata navarra acudía a educarse a prestigiosas universidades europeas como París, Toulouse, Montpellier y Bolonia (Tamburri Bariain, 2000, pp. 413-443). Sin 
(...) lo mas prouechossament que fazer se podra pora hutilidat et prouecho d'las dichas mis criaturas, et que partan los dineros de aquillos por cabeza, tanto l'uno como l'otro $(\ldots)^{28}$.

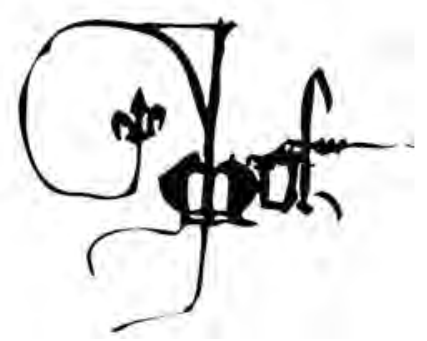

FIRMA AUTÓGRAFA DE JUAN MOLINER, 1399

3. EL TESTADOR: DIMENSIÓN ESPIRITUAL Y RELIGIOSA DE LAS ÚLTIMAS VOLUNTADES $^{29}$

La cercanía de la muerte obliga a Juan Moliner a testar — nadie mejor que él para saber su verdadero estado de salud-, manifestando no solo sus intenciones materiales, de las que ya se ha tratado, sino también las espirituales. Es Juan de Amicx, el joven, el notario que redactará el testamento del físico real, ateniéndose a formularios y modelos ya existentes ${ }^{30}$. Dicho testamento se inicia con la invocación a Dios y a la Virgen María - En el nonbre de Dios et de la Virgin Sancta Maria-, hecho bastante habitual en las últimas volunta-

embrago el caso de los médicos es un universo todavía por explorar. Un ejemplo de los intentos realizados para otros territorios hispanos, desde finales del siglo XV a mediados del siglo XVI, puede ser el estudio de Arrizabalaga, 2010, pp. 93-126.

28 La transmisión de libros relacionados con la medicina variaba según las circunstancias y las posibilidades económicas de sus dueños, como el traspaso intergeneracional de padres a hijos, o bien a familiares y conocidos, la compra o el robo en otros casos (Ferragud Domingo, 2005 , p. 104). En nuestro caso también se ha comprobado el préstamo de ejemplares por parte de los franciscanos estelleses.

29 Sobre esta cuestión sigo a Baldó Alcoz, García de la Borbolla y Pavón Benito, 2005, pp. 166-219.

30 Su padre ya aparece como notario entre la documentación conservada en el archivo de la parroquia de San Cernin o San Saturnino durante la primera mitad del siglo XIV (García Larragueta, 1976, p. 11). 
des de la época ${ }^{31}$. Evidentemente la validez del testamento queda corroborada por la buena salud mental del testador - estando en mi buena memoria et sano entendimiento - pese a la enfermedad, estableciendo su última voluntad frente a disposiciones anteriores - reuocando et anulando todos et quoalesquiere testamentes o codiçiellos fechos et ordenados por mi ata aqui-, con el fin de evitar malos entendidos en un futuro entre su mujer y sus hijos, sus principales beneficiarios. Finaliza el encabezamiento con el deseo de alcanzar la vida eterna - et la mi anima pueda yr a la gloria perdurable-, no como un mero preámbulo sino como manifestación de su piedad personal. De ahí, que la necesidad de plantear la posesión de las plenas facultades mentales que no sea un mero formulismo notarial para algunos autores (Baldó Alcoz, Gar-

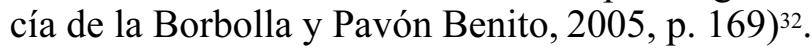

La primera alusión de Moliner, antes de dar paso a sus últimas voluntades, es reconocer de que tras su muerte el alma se separará de su cuerpo — quoando quiere que nostro seynnor Dios ordenare de mi anima que sailliere de mi cuerpo-, reconociendo implícitamente que anteriormente ambos estaban unidos (Pavón Benito y García de la Borbolla, 2007, p. 119) ${ }^{33}$. Su primera disposición es que su cuerpo sea trasladado a Pamplona, probablemente desde Olite, donde se encuentra al servicio del monarca y redacta su testamento, instando a sus cabezaleros que lo entierren en la iglesia de San Cernin de Pamplona,

en vna fuessa onesta, la quoal quiero et mando que sea conprada por mano de los dichos mis cabeçaleros de mis bienes, et que el dicho mi cuerpo sea sepelido onorablemente con torchas, çirios et otras candelas segunt a mi pertenesce et es vssado et costumbrado en la dicha ciudat de Pomplona en tales senblantes personas.

Esta práctica del ritual funerario establece la necesidad de determinar la posición social que el difunto disfrutaba en vida, quedando remarcada a tra-

31 La invocación dirigida en primer lugar hacia Dios Padre muestra una actitud de humildad y devoción con la que el testador acepta el inevitable tránsito de la muerte, disponiéndose a la voluntad del Creador. Por lo que respecta a la invocación a la Virgen María, ésta es la santa por excelencia. María es considerada la Madre de todos los fieles pero a la vez es Hija, representando un punto de unión entre Dios y lo hombres. Sobre todas estas cuestiones, véase Pavón Benito y García de la Borbolla, 2007, pp. 202 y 206-209. No obstante, todas estas fórmulas, desde un punto de vista bastante más prosaico que el religioso de estas autoras, bien pudieron ser utilizadas por los notarios con el fin de prolongar la extensión de los testamentos y así ampliar sus ganancias.

32 Puede consultarse también, Beroiz Lazcano, 2003, pp. 38-40.

33 No hay nada remarcable en esta idea que expone Juan Moliner, ya que refleja la antropología cristiana más tradicional sobre la vida y la muerte. 
vés de ciertas actitudes espirituales y distintos gestos externos propios de cada estamento (Baldó Alcoz, García de la Borbolla y Pavón Benito (2005), p. 174). De hecho las disposiciones sobre las luminarias, aunque vagas, dispuestas por Juan Moliner, están estrechamente relacionadas con su componente socioeconómico y su status ${ }^{34}$. A todo esto hay que señalar la elección del lugar de inhumación, la parroquia de San Cernin, una de las de mayor renombre de Pamplona ${ }^{35}$. Por lo que respecta a las mandas pías, Moliner establece que se realicen una serie de celebraciones litúrgicas, la novena ${ }^{36}$, trentena o treintena ${ }^{37}$ y cabo de año bien et onestamet segunt a mi pertenesce, además de una capellanía anual cantada,

con sus cirios et candelas, ofrenda de pan, començando luego como el mi cuerpo sera sepelido ata vn aynno en seguient et que el dicho cappeillan qui cantara o çelebrara la misa d'la dicha mi capeillania aynal aya de saillir sobre la mi fuessa en cada un dia a bendizirla segunt vssado et costunbrado es en la dicha eglesia, et la dicha mi muger aya de leuar a ofreçer et ofrezca las dichas ofrenda de pan et candelas en la dicha mi aynal.

La última disposición de tipo espiritual es la obligación de que los albaceas - su mujer, Sancho de Aoiz, abad de Urroz, Sancho de Oteiza, abad de Aibar, y Pedro García de Eguillor, notario, los dos últimos secretarios del rey- o sus sucesores dispongan que en caso de que sus descendientes murieran sin sucesión, utilicen las rentas de los bienes que ha dispuesto en el mayorazgo para sus hijos, con el fin de financiar, ya se a dicho anteriormente, otra capellanía cantada anual perpetua simple por su alma, la de su mujer et criaturas et de mis mayores et por las animas a qui yo so tenido rogar; ejemplo de intentar perpetuar su memoria y la de sus familiares al mismo tiempo que

34 Sobre estos aspectos, véase Baldó Alcoz, 2002, pp. 197-210 y 2006a, pp. 385-402.

35 De hecho en esta parroquia estába instaurada la cofradía de Santa Catalina, una de las más importantes de Pamplona, de la que era cofrade el propio rey Carlos III. Las referencias a 'fosas' suelen ser más abundantes en el medio rural, mientras que en el urbano resulta más corriente mencionar la palabra 'sepultura', aunque no parece que el formato variara esencialmente (Baldó Alcoz, García de la Borbolla y Pavón Benito, 2005, p. 197).

36 Era la celebración de una misa por el alma del difunto durante los nueve días que seguían al funeral. Sobre este tipo de misas, véase Baldó Alcoz, 2006b, pp. 356-359.

37 La trentena gregoriana comprende un conjunto de 30 misas bajas celebradas a razón de una por día consecutivo o bien en el mismo día (Baldó Alcoz, García de la Borbolla y Pavón Benito, 2005, p. 205). Su propósito era facilitar alivio a los padecimientos sufridos por el alma del difunto, durante su estancia en el Purgatorio expiando sus pecados. Sobre las variedades de estas misas, véase Baldó Alcoz, 2006b, pp. 359-367. 
la salvación de sus almas ${ }^{38}$. De diciembre de 1479 es el acuerdo entre los obreros, clérigos y vicario de San Cernin con los cabezaleros de Juan de Itúrbide por el que

queriendo complir las clausulas de los dichos testamentos, assi de dicho maestre Johan Moliner como del dicho Martin d'Iturbide, su yerno, qui fueron, (...) con liçençia, permisso e autoridat del sennyor official de Santa Maria de Pamplona, por virtut de las sobre dichas clausulas de los dichos testamentos (...) vistas y examinadas, por quanto los dichos bienes non vastan para la fundacion de la capellania que el dicho maestre Johan Moliner por su testamento mandaua fundar. Ouido deliueracion e maduro conssejo con hombres letrados y entendidos acerqua desto a seruicio de Dios e de la gloriosa Virgen (...), los dichos Johan Loppiz e Sancho de Yrigoyen, cabeçaleros subrogados e substituydos del dicho Martin d'Iturbide qui fue, (...) fundaron e ordenaron desde agora por las presentes para en todos tiempos a perpetuo quoatro aniuerssarios con sus vigilias e nuebe leçiones de muertos e con missa solempne de Requiem con diacono y subdiacono, que sean dichas e çelebradas en la dicha yglesia de seynnor Sant Çernin cada annyo en el altar mayor de la dicha yglesia a perpetuo. Es a ssauer, la primera missa del aniuerssario el viernes de las quoatro tiemporas empues el primer dia de quoaresma, la otra el viernes de quoatro tiemporas de la pentecosta, la terçera el viernes de las quoatro tiemporas de Santa Cruz del mes de septiembre e la quoatrena el viernes de las quoatro tiemporas empues Santa Luçia $(. . .)^{39}$.

38 Sobre estas cuestiones, véase Pavón Benito y García de la Borbolla, 2007, pp. 260-267.

39 En cada una de dichas misas el vicario y los clérigos de San Cernin debían de salir con la cruz sobre la sepultura del dicho Martin d'Iturbide donde esta sepellido (...) e diran hun ressponsso con su oraçion de mortuys, segunt es vsado e acostumbrado en la dicha yglesia de Sant Cernin cada annyo a perpetuo. El rezo del responso sobre la tumba del difunto parece ser una práctica habitual (Baldó Alcoz, García de la Borbolla y Pavón Benito, 2005, p. 211). Para pagar estas misas se ofrecieron 12 libras carlines anuales, pertenecientes a las rentas de las sobre dichas casas que fueron del dicho maestre Johan Moliner, situadas en la dicha Rua de la Correyeria del burgo de la dicha çiudad (...). Item mas, vna pieça de tierra de seze rouadas poco mas o menos, situada en el termino de Ychastia, affrontada d'una part con pieza de Johan d'Esparça, mercadero, e de la otra part con pieza de Anchote de Lauayen, pelegero, e de Martin d'Arrieta, çapatero, e de la otra con el camino sendero que van de Pamplona enta Sant Miguel de Baraniany (sic). Item otra pieça de doze rouadas poco mas o menos situada en Cascalleta, affrontada d'una part con pieza del concejo de Sanssoany (sic) e de la otra con vina de Sanchot de Yçall, calderero, e de las otras dos partes con los caminos publicos (...). Item otra pieza de seze robadas poco mas o menos situada en Lezcayru (...). Item vna pieza en el termino de Mendilorri clamado Sayquin (...). Item otra pieza a mesmo affrontada con vina de Loppe de Mendilorri e de las otras otras dos partes con vinas de Garcia Martinez d'Abiçu, escudero. Item otra pieza en el termino de Mutiloa de Suso, clamado Sayquin (...). Item otra pieza de seze robadas en el termino de de Mutiloa de Iuso (...). Item vna pieça ay luego de doze robadas affrontada d'una part con sendero vezinal e de las otras dos partes 


\section{CONSIDERACIONES FINALES}

La definición del testamento medieval como el documento mediante el cual el fiel cristiano proyectaba los beneficios futuros para su alma, tal y como proponen algunos autores (Pavón Benito y García de la Borbolla, 2007, p. 270), resulta una fuente de primera mano como acto de piedad y de fe además de permitir llegar a conocer aspectos materiales de aquellos quienes los redac$\operatorname{taron}^{40}$. Este tipo de documentación adquiere todavía más importancia cuando la escasez de datos sobre los testadores hace que se pueda acceder a información para el cocimiento de su periplo vital — situación socioeconómica, descendencia y otras cuestiones referentes a la vida privada del testador- no recogida en otra clase de documentación.

El testamento de Juan Moliner, médico de los dos monarcas navarros más importantes de la dinastía Evreux, aporta una visión más humana de lo que las fuentes contables nos proporcionan, hasta el momento el principal material utilizado para seguir sus andanzas al servicio de los reyes de Navarra. De procedencia desconocida, aunque probablemente de origen francés/gascón aludido como Jehan Moulinier, en algún documento escrito en romance francés-, si nos atenemos a la flor de lis que decora su firma autógrafa y a los nombres de sus hijos varones, comenzó a prestar sus servicios a partir del último cuarto del siglo XIV durante poco más de 25 años ${ }^{41}$.

De un nivel de vida más o menos desahogado, mantuvo relaciones, muchas de ellas probablemente facilitadas por su oficio médico y por su vinculación con la corte, no solo con los monarcas sino también con personajes de un status social similar al suyo. Lo dicho viene a confirmarse al comprobar en el testamento sus

con pieça e vina de la abadia del dicho lugar de Mutiloa pertenecientes a dicho Juan Moliner, Juan de Itúrbide y Juana Moliner mientras biuian, auian e tenian (...) (APSS, caj. 2, Pergaminos, $\mathrm{n}^{\circ} 33$, sec. I, $n^{\circ}$ ant. 64 (32)). Distintos ejemplos de 'memoria funeraria' a familiares y parientes, en Pavón Benito y García de la Borbolla, 2007, pp. 260-265.

40 En palabras de Ferragud Domingo (2005, p. 605) el testamento «es un claro indicador de la situación económica y social del que dicta. Ambas circunstancias son producto de la noble naturaleza del testamento como pasaporte al más allá: la religiosa (garantizar la vida eterna) y la jurídica (ordenación del patrimonio acumulado en vida)».

41 En la ciudad de Valencia se ha documentado la existencia de unos Moliner, a finales del siglo XIV, dedicados a la sanidad, como el cirujano Francesc Moliner, alias Metge, y Pere Moliner, alias Metge (Ferragud Domingo, 2005, p. 153). No obstante, pese a la falta de datos, los indicios parecen sugerir como más probable que Juan Moliner fuera originario del otro lado de los Pirineos que de la Corona de Aragón. Si existe la posibilidad de algún tipo de vínculo familiar entre ellos, se desconoce por el momento. 
deudores, sus tierras, la ubicación de la casa donde vive su familia y la elección de la parroquia para su enterramiento. Una familia ampliada con personajes que no poseen un vínculo sanguíneo con ellos (sirvientes o criados), y que a falta de una estrecha relación paterno-filial de afecto mutuo debido a la poca edad de los hijos, esta afectividad queda demostrada especialmente con alguno de esos sirvientes. De hecho estos personajes eran considerados como unos miembros más de la familia. A esto hay que sumar la especial relación que parece mantener con su confesor, el franciscano fray Pedro de Eza, a la sazón confesor de la reina Leonor de Trastámara, con el abad de Urroz, y con Sancho de Oteiza y Pedro García de Eguillor, estos dos últimos secretarios del rey, a quienes junto con su mujer les nombró albaceas de sus últimas voluntades.

El respeto y consideración que Juan Moliner alcanzó en vida quedan también demostrados tras su muerte en la persona de su yerno, al que no conoció, el notario de la Cámara de Comptos Juan de Itúrbide. Por distintas vicisitudes, como se ha podido comprobar, la herencia del difunto físico quedó sin herederos responsables por lo que Itúrbide en su testamento ordenó cumplir las mandas pías que su suegro dejó por si ocurría el caso. Así, setenta y seis años después de la muerte de Juan Moliner y veintidós de la de Juan de Itúrbide los albaceas de este último acuerdan con los representantes de la parroquia de San Cernin cumplir, en la medida que el dinero de ambas herencias permite, las últimas voluntades de los dos difuntos.

La manera de promocionar socialmente por parte de Juan Moliner, al igual que se ha venido confirmando para otros territorios hispanos, debió de ser a través de su matrimonio y su educación, y de hecho lo consiguió, no en vano fue físico del rey durante muchos años, pese a las lagunas que sobre esos dos aspectos tenemos. Una dignificación social que queda también recogida en sus últimas voluntades en lo referente a su dimensión espiritual tanto por la elección de una fosa honesta como por su deseo de que su cuerpo sea enterrado con antorchas, cirios y otras candelas según a su estado social tal y como se acostumbraba en Pamplona. A esto hay que añadir todas las mandas pías que propone se realicen para la salvación de su alma, aunque pese a la importancia del testador éste no hace ostentación excesiva en comparación con otros testamentos de la época. 


\section{BIBLIOGRAFÍA}

Arrizabalaga, Jon (2002), "Francisco López de Villalobos (c. 1473-c. 1549), médico cortesano", Dynamis. Acta Hisp. Med. Sci. Hist. Illus., 22, 2002, pp. 29-58, p. 34.

Arrizabalaga, Jon (2010), "Spanish Medical Students' peregrinatio to Italian Universities in the Renaissance". En: Grell, Ole Peter; Cunningham, Andrew; Arrizabalaga, Jon (eds.), Centre of Medical Excellence? Medical Travel and Education in Europe, 1500-1789, Farnham-Burlington, Ashgate, pp. 93-126.

Arrizabalaga, Jon (2012), "Asistencia, caridad y nueva ética de la responsabilidad colectiva ante la salud y la pobreza en el espacio urbano occidental del Antiguo Régimen". En: Huget-Termes, Teresa; Verdés, Pere; Arrizabalaga, Jon; Sánchez Martínez, Manuel (eds.), Ciudad y hospital en el occidente europeo, 1300-1700, Lleida, Milenio, (en prensa).

Baldó Alcoz, Julia (2002), "Un aspecto de los funerales a través de la legislación civil en la Navarra bajomedieval: el uso de antorchas durante el cortejo". En: Erro Gasca, Carmen; Mugueta Moreno, Íñigo (eds.), Grupos Sociales en la historia de Navarra: relaciones y derechos (Actas del v Congreso de Historia de Navarra, Pamplona, septiembre de 2002), vol. 2, Pamplona, Sociedad de Estudios Históricos de Navarra, pp. 197-210.

Baldó Alcoz, Julia (2005), Réquiem aeternam. Ritos, actitudes y espacios en torno a la muerte en la Navarra bajo medieval, Pamplona, Universidad Pública de Navarra (tesis doctoral inédita).

Baldó Alcoz, Julia (2006a), "Segunt a mi estado fazer pertenesce. Imagen y memoria de los grupos sociales privilegiados en la Navarra bajomedieval: el cortejo funerario". En: Navarra: Memoria e imagen (Actas del vi Congreso de Historia de Navarra, Pamplona, septiembre de 2006), vol. 2, Pamplona, Sociedad de Estudios Históricos de Navarra, pp. 385-402.

Baldó Alcoz, Julia (2006b), "Las misas post mortem: simbolismos y devociones en torno a la muerte y el más allá en la Navarra bajomedieval", Zainak, 28, pp. 353-374, pp. 356-359, 359-367.

Baldó Alcoz, Julia; García de la Borbolla, Ángeles; Pavón Benito, Julia (2005), "Registrar la muerte (1381-1512). Un análisis de testamentos y mandas pías contenidos en los protocolos notariales navarros", Hispania. Revista de Historia, 219, pp. 155-226, pp. 116-219, 169, 174, 197, 205, 211, 208, 210.

Beroiz Lazcano, Marcelino (2003), "La mort dans les terres du Sud: rites funéraires en Navarre au Moyen Âge (xiii $-\mathrm{xv}^{\mathrm{e}}$ siècles)". En: Lemaitre, Nicole (ed.), Religión et politique dans les sociétés du Midi (126 Congrès Nacional des Sociétés Historiques et Escientifiques, Toulouse, 2001), París, CTHS, pp. 37-48, pp. 38-40.

Cabré i Pairet, Montserrat; Salmón Múñiz, Fernando (2001), "Poder académico versus autoridad femenina: la Facultad de Medicina de París contra Jacoba Félicié (1322)". En Cabré i Pairet, Montserrat; Ortiz Gómez, Teresa (coords.), Sanadoras, matronas y médicas en Europa: siglos xii-xx, Barcelona, Icaria, pp. 55-76.

Ferragud Domingo, Carmel (2005), Medicina i promoció social a la Baixa Edat Mitjana (Corona d'Aragó, 1350-1410), Madrid, Consejo Superior de Investigaciones Científicas, pp. 104, 605, 153. 
García Ballester, Luis (2001), La búsqueda de la salud. Sanadores y enfermos en la España medieval, Barcelona, Península, pp. 213-225.

García Larragueta, Santos (1976), Archivo parroquial de San Cernin de Pamplona. Colección hasta 1400, Pamplona, Diputación Foral de Navarra-Institución Príncipe de Viana, p. 11.

Gran Enciclopedia de Navarra, t. viii, Pamplona, Caja de Ahorros de Navarra, 1990, p. 10.

Green, Monica (1989), "Women's Medical Practice and Health Care in Medieval Europe", Sings. Journal of Women in Culture and Society, 14, pp. 434-473, p. 447.

López Gómez, José Manuel (2010), "Mujer y medicina en el Burgos bajomedieval: Mencía González, 'física' de Aranda de Duero (1495)", Boletín de la Institución Fernán González, 241, pp. 215-233.

Marcotegui Barber, Beatriz (2009), "Instructio morum et fidei”. La predicación en el reino de Navarra en el siglo xv, Pamplona, Gobierno de Navarra, p. 92.

Martinena Ruiz, Juan José (1974), La Pamplona de los Burgos y su evolución urbana (Siglos xii-xvi), Pamplona, Diputación Foral de Navarra-Institución Príncipe de Viana-Consejo Superior de Investigaciones Científicas, p. 207.

Martínez Crespo, Alicia (1994), "Mujer y medicina en la Baja Edad Media", Hispania. Revista de Historia, 186, pp. 37-52, pp. 44-45.

McVaugh, Michael R (1993), Medicine befote the plague. Practitioners and their patients in the Crown of Aragon, 1285-1345, Cambridge, Cambridge University Press, pp. 103-107.

Narbona Cárceles, María (2006), La corte de Carlos III 'el Noble', rey de Navarra: espacio doméstico y escenario del poder, 1376-1415, Pamplona, Eunsa, p. 255.

Noáin Irisarri, José Joaquín (2003), "Estrategias económico-familiares de la nobleza media de Navarra en la Edad Moderna (1500-1700)”. En: Fernández Romero, Cayetano; Moreno Almárcegui, Antonio (eds.), Familia y cambio social en Navarra y País Vasco. Siglos xiii-xx (Simposio de Historia de la Familia, Pamplona, 20 y 21 de septiembre de 2002), Pamplona, Instituto de Ciencias para la Familia, pp. 91-134, pp. 9396, 97-98.

Pavón Benito, Julia; García de la Borbolla, Ángeles (2007), Morir en la Edad Media. La muerte en la Navarra medieval, Valencia, Universidad de Valencia, pp. 265-266, 202, 206-209, 119, 260-267, 270, 260-265.

Ramírez Vaquero, Eloísa (1990), Solidaridades nobiliarias y conflictos políticos en $\mathrm{Na}$ varra, 1387-1464, Pamplona, Gobierno de Navarra, p. 114.

Salmón Múñiz, Fernando (1997), "La historia de las mujeres en la práctica de la Historia de la Medicina". En: Segura Graíño, Cristina (coord.), La historia de las mujeres en el nuevo paradigma de la historia, Madrid, Asociación Cultural Al-Mudayna, pp. 37-54, pp. 38-43.

Serrano Larráyoz, Fernando (2004), Medicina y enfermedad en la corte de Carlos III 'el Noble' de Navarra (1387-1425), Pamplona, Gobierno de Navarra, pp. 113,132, 78, $55,89,192$.

Serrano Larráyoz, Fernando (2009), “Astrólogos y astrología al servicio de la monarquía navarra durante la Baja Edad Media”, Anuario de Estudios Medievales, 39 (2), pp. 539-554, pp. 548-549. 
Serrano Larráyoz, Fernando (2011), "Boticarios en el reino de Navarra durante la Baja Edad Media: una aproximación prosopográfica”, en González Bueno, Antonio; López Andújar, Guillermina; Cabezas López, María Dolores; Martín Martín, Carmen; Esteva de Sagrera, Juan (eds.), Homenaje al Prf. Dr. José Luis Valverde, Granada, Sociedad de Docentes Universitarios de Historia de la Farmacia de España-Universidad de Granada, pp. 263-326, pp. 278-279.

Tamburri Bariain, Pascual (2000), "Navarra en el sistema europeo medieval". En: de la Iglesia Duarte, José-Ignacio (coord.), La enseñanza en la Edad Media (Actas de la x Semana de Estudios Medievales, Nájera, del 2 al 6 de agosto de 1999), Logroño, Instituto de Estudios Riojanos, pp. 413-443. 
Olite, 13, febrero, 1403

Testamento de Juan Moliner, fisico del rey Carlos III el 'Noble' de Navarra Archivo Parroquial de San Saturnino, caja 1, Pergaminos, $n^{\circ}$ 7, sec. I, $n^{\circ}$ ant. 34 (8)

En el nonbre de Dios et de la Virgin Sancta Maria. Seppan quantos esta present carta veran et oyran que como yo Johan Moliner, maestro en medicina et fisigo del muy et inclito seynnor mi seynnor el rey de Nauarra, estando / en mi buena memoria et sano entendimiento maguer enfermo en mi persona, fago et ordeno este mi vltimo et postremero testament, seyendo present et laudant a las cosas infrascriptas Gracia d'Urssua, mi muger, reuocan- / do et anulando todos et quoalesquiere testamentes (sic) o codiçiellos fechos et ordenados por mi ata aqui, et por tal que enpues mis dias yra, debbat et contienda no aya de concester ( $\mathrm{sic}$ ) entre la dicha mi muger et mis criaturas / et la mi anima pueda yr a la gloria perdurable amen.

Primerament ordeno et mando que quoando quiere que nostro seynnor Dios ordenare de mi anima que sailliere de mi cuerpo quiero, mando et me plaze que los mis cabeçaleros / de iuso scriptos o los qui mas curosos et diligentes seran, que mi cuerpo fagan leuar ondradament a la ciudat de Pomplona a las mis casas, et en seguient, quando a eillos sera bien visto et fuere ora conpetent, que / el dicho mi cuerpo fagan enterrar et sepelir en la egllesia (sic) parrochial de seynnor Sant Cerni en vna fuessa onesta, la quoal quiero et mando que sea conprada por mano de los dichos mis cabeçaleros de mis bienes, et que / el dicho mi cuerpo sea sepelido onorablement con torchas, çirios et otras candelas segunt a mi pertenesce et es vssado et costumbrado en la dicha ciudat de ${ }^{42}$ Pomplona en tales et senblantes personas.

Item mando que sea / fecha la mi nouena, trentenario et cabo d'aynno bien et onestament segunt a mi pertenesce por mano de mis cabeçaleros o de quoalquiere d'ellos.

Item mando, quiero et me plaze que sea cantada vna capeillania aynal en la / dicha eglesia de seynnor Sant Çerni con sus cirios et candelas, ofrenda de pan, començando luego como el mi cuerpo sera sepelido ata vn aynno en seguient et que el dicho cappeillan qui cantara o çelebrara / la misa d'la dicha mi capeillania aynal aya de saillir sobre la mi fuessa en cada un dia a bendizirla segunt vssado et costunbrado es en la dicha eglesia, et la dicha mi muger aya de leuar a ofreçer et ofrezca / las dichas ofrenda de pan et candelas en la dicha mi aynal.

Item mando et ordeno que todo que queriant que paresciere por buena uerdat que yo sea tenido en cosa alguna que los mis cabeçaleros de iusso scriptos o los que mas curosos / et diligentes seran, fagan emienda et satisfacion de mis bienes conoscidas deuidamente las dichas deudas que yo tenido sere:

Item heredo en vez de heredar et mueble a Peyreton, mi fijo et d'la dicha Gracia, mi muger, segunt / fuero, en vna arincada de vina en la vina que yo he en Cascailleta, termino de Pomplona, la quoal se afruenta d'una part con pieça del conçeillo de Sanssoain et de la otra part con pieça de dona Miquela, vezina de Pomplona que fue, / et mas en 20 sueldos carlines por fazer de aqueillos todas sus propias voluntades.

$$
42 \text { de] Ms. repetido. }
$$


Item assi bien en vez de heredar et mueble heredo a Lancalot, mi fijo et de la dicha Gracia mi muger, en vna arincada de vina en la vina que yo / he en Mutiloa, la quoal se afruenta d'una part con vina de Sancho d'Erro, podador, vezino de Pomplona, et d'la [otr]a part con vina de la abbadia de Muttiloa, et mas en 20 sueldos carlines pora fazer de aqueillos todas sus propias / voluntades.

Item assi bien en vez de hedar (sic) et mueble heredo, segunt fuero, a Johana, mi fija et d'la dicha Gracia, mi muger, en vna arinçada de vina en la vina que yo he en el termino de Sayquin, la quoal se afruenta d'una / part con vinas de Garcia Martiniz d'Arbizu, escudero, d'anbas partes, et mas en 20 sueldos carlines pora fazer de aqueillos todas sus propias voluntades.

Item estas son las deudas que me deuen en Navarra las que a present me mienbra? / et aqueillas quiero et mando que sean cobradas por mano de mis cabeçaleros o de aqueillos qui mas curossos sean pora conplir este mi testament:

Primero me deue Pedro d'la Plana, molinero, vezino de Tudela, dozientas libras / febles poco mas o menos. Item me deue Martin Yuañes de Los Arquos, notario qui fue, dos escudos d'oro. Item Miguel d'Egueras me deue dos escudos d'oro que le preste. Item me deue Pascoal de Licarraga, mercadero, vezino d'Estella, seysçientas / libras febles poco mas o menos. Item me deue Johan de Sangüessa, notario d'la Cort, vezino d'Estella, dozientas et 20 libras fuertes, en pero d'esta suma he tomado cierta quoantia de dineros et li sean rebatidos d'la dicha suma / segunt que se trobara por reconoscimientos que eill tiene de mi. Item me deue Tronchet, scudero, vn escudo. Item su hermano maestre Frances vn escudo. Item mas me deuen los herederos et bienes de Johan Garcia de Beunça que fue / bien la montança de dozientos florines d'oro d'Aragon, en pero esto lexo a la discrecion de Gracia, mi muger, et de Enecot, mi moço, que eillos ayan de contar con los herederos del dicho Johan Garçia o con aqueillos a qui pertenezca. Item / mando et lexo que los mis cabeçaleros de iuso contenidos o quoalquiere d'ellos qui mas curossos et diligentes seran ayan poder de demandar auer et cobrar las sobredichas mis deudas et otras que se trobaran por buena verdat que seran / a mi, maguer en este mi present testament non son nonbradas ni espaçificadas (sic). Item me tiene Johan Crozat, chinberlinc, dos taças de plata marcales, enpeynnos de 20 libras febles, et que mis cabeçaleros las quiten de mis / bienes. Item me tiene Pere d'Azedo, espeçiero, vezino de Pomplona, vna taça de plata, marcal por siete libras fuertes, asi bien que sea cobrada et quitada por los dichos mis cabeçaleros de mis bienes. Item me tiene la muger de Pere d'Arci / dos taças de plata et que los dichos mis cabeçaleros quiero et mando que las cobren. Item me tiene Johan d'Izco, mercadero, vezino de Pomplona, vna taça de plata marcal, enpeynnos, et que eill en su conçiençia diga per quanto esta en- / garada, et que sea quitada por mano de mis cabeçaleros o de quoalquiere d'ellos de mis bienes.

Item instituyo, ordeno, quiero et mando que Gracia, mi muger, sea et aya de seer tutriz testamental d'las dichas mis criaturas et suyas, et / casando o non casando que tienga et aya de tener en toda la su vida todos mis bienes muebles et heredades et suyos que eilla et yo auemos, et son estos que se siguen: Primerament, la mi casa que yo he en la Rua Maor d'la Correyeria / de Pomplona con su mueble, la quoal se afruenta d'una part con casas de Martin Bertran, mercadero, vezino de Pomplona, et d'la otra part con casas de Miguel d'Eguaras, correyero, vezino de Pomplona, et todos et quoalesquiere otros bienes 
muebles, / heredamientos pertenescientes a mi, asi como casas, casales, piecas, vinas, huertos, oliuares, prados et vezindades o qualesquiere otros bienes, heredameintos a mi en todo el regno de Nauarra o en quoalquiere otra tierras et seynnorios sean / et puedan ser et pertenescer por quoalquiere titulo o razon sean, quiero, mando et lexo a la dicha Gracia, mi muger, que las tenga et se goze et esploite de aqueillas casando o non casando en toda la su vida, como dicho es de partes de suso. / Enpero aqueillos meiorando et non apeorando nin vendiendo nin agenando cosa alguna en su vida ni enpues sus dias por eilla nin otro por eilla saluo su mitat del mueble, et que la dicha Gracia sea tenida criar a las dichas nuestras criaturas de comer, / beuer, vestir et calçar [a]ta tanto que ayan tiempo de 20 aynnos cada una d'ellas, et que a los fijos faga mostrar en la escuela a leyer et screuir bien et suficiement a su leal poder et a la fija que le mostre buenas costumbres, et que las regezca co- / mo tutriz et madre, conseiando et criandolas bien et onestament.

Et de que sera finado la dicha Gracia, mi muger, luego enpues sus dias ordeno, mando que la dicha casa con la mitat del mueble por mi part herede et aya de heredar con todas las / sobredichas heredades que yo he et a mi pueden auer et pertenescer segunt que de partes de suso dicho es, el dicho Peyreton, mi fijo primogenito et de la dicha Gracia, mi muger, et que eill las tenga, espleite en toda la su vida, non vendiendo ni / agenando de aqueillas cosa alguna, ante quiero et mando que vayan de mayorio en mayorio en las dichas mis tres criaturas et de su genoyla et generation, non vendiendo ninguna d'ellas cosa alguna de los dichos mis bienes, casas, / heredamientos, eredando l'uno por muert del otro por herencia legitima et mayorio segunt fuero de Nauarra. Et en caso que el dicho Peyreton, mi fijo, en su vida o su criazon enpues su muert o el dicho Lançalot, mi fijo, enpues / sus dias o su criazon enpues eill [o la] dicha Johana, mi fija, o sus criazones o quoalquiere d'ellos o de sus genoillas, qui las dichas casas con las dichas heredades teniere por herencia legitima de mayorio como dicho es, ninguno d'eillos fuese / loco, gastador o de mal regimiento o gouernamiento que los mis cabeçaleros de iuso scriptos o quoalquiere d'ellos o el subrrogado o subrrogados por eillos ayan poder de meter sobre eillos o quoalquiere d'ellos qui la dicha mayoria tenian / et heredaria tutor et goardador de los dichos bienes, casas et heredamientos por mi limitados de partes de suso al sobredicho mayorio, et esto segunt que el seynnor rey o la su noble Cort le auriria de fazer por tal que los dichos mis bienes, / heredamientos, si deuenia d'las dichas mis criaturas sen criazones o las criazones sin criazones puedan ser obseruados et goardados a la disposicion que yo de partes de iuso ordenare et dispondre de aqueillos.

Item si deueria de los dichos mis fijos / et fija sin criaturas o las criaturas sin criaturas, lo que Dios non mande, ordeno mando et lexo que los mis cabeçaleros de iuso contenidos o qualquiere d'ellos, o el subrrogado o subrrogados, por eillos ayan de poner sobre las dichas mis / casas, piecas, vinas, huertos, oliuares et sobre los otros bienes, heredamientos que yo he designado en el dicho mayorio a las dichas mis criaturas vna capeyllania aynal perpetua simple, la quoal quiero que sea cantada en la dicha eglesia / de Sant Çerni por mi anima, d'la dicha mi muger et criaturas et de mis mayores et por las animas a qui yo so tenido rogar, et que esta se cante con las rentas que podran saillir de las dichas mis casas, heredades, las que yo he designado / al dicho mayorio.

Item mando et lexo a Marito, mi moca, pora si et pora su casamiento los dineros que me deue el arcidiagno de val d'Ayuarr qui a present es, que son 80 libras fuertes, los 
quoales eill me los tiene d'la mi pension por el / mi benefiçio deuido por los prior, capitol et canonigos de Pomplona.

Item mas li dexo a la dicha Mariton 10 cafices de trigo. Item mas li lexo a la dicha Mariton seys cargas de vino mosto del meior de mi casa. Item lexo a Enecot, / mi moço et seruidor mio, mi mula morena que yo solia caualgar ante desta mi enfermedat pora si et fazer todas sus propias voluntades d'eilla, et mando mas que el dicho Enecot more et viua en toda la su vida en mi casa de Pomplona, / comiendo et beuiendo de lo que en casa se trobara comunlment (sic), et asi bien que aya de auer a bien vista d'la dicha Gracia, mi muger, su bestir sufiçient segunt la facultat vista entre eilla et eill sera, et le acomendo al dicho Enecot las dichas mis criatu- / ras que la regesta segunt fuesen suyas propias, et le encargo en la su anima et conciencia que renda a las dichas mis criaturas si algunas cosas en los tiempos pasados a tomado o apartado en los tiempos pasados de mis bienes. Item mas / mando et lexo a la dicha Johana, mi fija, lo que me deuen en las montaynnas de Nauarra por pan, vino et otras cosas que yo preste a otros por mi de mis bienes, que pueden ser bien ata la montança de cient libras carlines, poco mas o menos, / et esto con boluntat de la dicha Gracia, mi muger, et que estos dineros sean puestos luego como seran cobrados por mano de los dichos mis cabeçaleros o de quoalquiere d'ellos en vna buena persona en Pomplona a vtilidat, prouecho d'la dicha Johana, mi fija, ata / el tiempo del su casamiento, et pora quoando a eilla sera neçessario et que entonz le sean librados et dados. Item mando et lexo a la dicha Gracia, mi muger, todas mis ropas de vestir pora fazer todas sus propias voluntades, exceptado que lexo al dicho Enecot, mi moço, / mi opa de peis sen la forradura et vn capirot de los mios, el meior pora (sic). Item mando a mi moçet, Micheto, que sean dados de mis bienes tres florines d'oro. Item deuo prender en el arçidiagnado tres cafizes et medio ${ }^{43}$ de trigo, et quiero que sean dados a Garcia, / mi moço. Item mando et lexo a Lançalot, mi fijo, cient libras carlines pora en su casamiento al tiempo que seran cobrados las sobredichas mis deudas, et que aqueillas le sean puestas por mano de los dichos mis cabeçaleros o de quoalquiere d'llos en poder de / vna buena persona en comienda a vtilidat et prouecho del dicho Lançalot, mi fijo. Item mando et lexo al dicho Peyreton, mi fijo primogenito, todos mis libros de medicina que yo a present he en Navarra et quoalesquiere otros libros que yo he de / ciencia, exceptado que si libros ningunos se trobaren entre aqueillos de nigromancia que aqueillos libros de nigromancia sean quemados por mano de mis cabeçaleros de iuso scriptos, et los otros sobredichos libros sean dados et librados al dicho Peyreton, / mi fijo, pora que eill aprenga d'la ciencia que yo aprisi en aqueillos. Et en caso que el dicho Peyreton, mi fijo, non quisiere aprender en la dicha ciencia de los dichos mis libros que aqueillos sean dados et librados al dicho Lancalot, mi fijo, por tal que eill aprenga / en aqueillos d'la sobredicha ciencia. Et en casso que los sobredichos Peyreton et Lancalot, mis fijos, non quisiesen estudiar ni aprender en las sciencias que son contenidas en los dichos mis libros, nin conseguir nin trauajar en aqueillos mando que los dichos / mis libros tenga en deposito et goarda la dicha mi muger et madre d'las dichas mis criaturas, et que aqueillos sean vendidos lo mas prouechossament que fazer se podra pora hutilidat et prouecho d'las dichas mis criaturas, 
et que partan los dineros de aqui- / llos por cabeza, tanto l'uno como l'otro. Item mando que sean rendidos dos libros que yo tengo de los predigadores d'Estella, de tablas vieias, que es el vno el Albert Maor et el otro Fisicas. Item lexo a mi confessor don fray Pere d'Eça quoatro florines d'oro / d'Aragon por tal que mi anima aya en comienda. Otrossi yo el dicho Johan Moliner, maestro et testador sobredicho, fiando d'la grant discrecion et buena fama, lealdat et verdat que en eillos es, et entendiendo que eillos me amauan en mi vida / amorablement et lealment et que enpues mis dias amaran a mi anima, fago, instituyo et establesco por mis cabeçaleros et executores d'este mi vltimo testament et vltima voluntat a los honrrados et discretos don Sancho d'Aoiz, abbat d'Urroz, / et a la dicha Gracia, mi muger, qui estan presentes, et por delant a don Sancho d'Otheica, abbat de Ayuar et a Pero Garcia d'Eguirior, notarios et secretarios del dicho seynnor rey, absentes. A todos ensenble et a cada uno dellos por si et por el todo pora / conplescer este dicho mi testament de mis bienes et pora demandar, auer et cobrar las sobredichas mis deudas et de aqueillas complescer et fazer complescer este dicho mi testament, et pora demandar aqueillas ante quoalquiere juge ordinario, ecclesiastico et / seglar, et en casso que d'las dichas mis deudas se detard[a]s largament de cobrar que la dicha mi capeylania non çesse de se çelebrar nin la ofrienda de aqueilla con sus nouena, trentena et cabo d'aynno, et que todo esto sea pagado del pan, vino et / otras cosas que yo tengo et sabe la dicha mi muger en mi poder, et que de aqueillo se fornezqua a bien vista de los dichos mis cabecaleros sen que mal preuyzio nin daynno non venga a las dichas mi muger et criaturas. Et nos los dichos Sancho d'Aoiz, abbat / d'Urroz, et Gracia d'Urssua, muger de vos el dicho maestre Johan Moliner, testador sobredicho, qui presentes somos a la factura deste present testament a la rogaria de vos el dicho maestre Johan, por tales cabeçaleros nos otorgamos et luego de present / nos encargamos de la execucion d'eill, et pora fazer conplir las cosas contenidas en este present testament. Et yo la dicha Gracia d'Urssua, muger de vos el dicho maestre Johan Moliner, mi marido, leho, aprouo, ratifico este present testament de punto en punto / segunt que por el dicho mi marido ha seido dispuesto et ordenado de partes de suso, et prometo de non venir en contra en ren nin en partida de aqueill. Desto son testigos qui clamados et rogados fueron a todas las cosas sobredichas et qui por tales / testigos se otorgaron nonbradamente los honorables et discretos don fray Pere d'Eça, confessor de la seynnora reynna de Nauarra, et don fray Garcia de Olit, freyres d'la Orden de Sant Francisco. Esto fue fecho en la villa / de Oilit, XIII ${ }^{\circ}$ dia del mes de febrero Anno Anativitate Domini Millesimo Quadrigentesimo Terçio./

(Signo) Et yo Johan d'Amicx, el joven, por autoridat appostolica de la Cort Maor et por todo el regno de Nauarra notario, qui a todas las cosas sobredichas et cada una d'llas fuy present, et a instançia et requisicion / del sobredicho maestre Johan, testador sobredicho, de la nota original por mi reçebida en la forma et manera sobredicha con mi propia mano scriui et fiz en eilla este mi signo acostumbrado en / testimonio de verdat.

Recibido: 2 de marzo de 2011

Aceptado: 12 de noviembre de 2011 